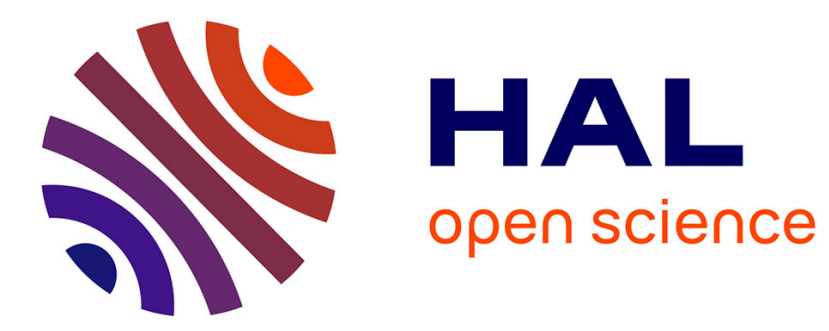

\title{
MULTILAYERS AND LOW ANGLE NEUTRON SCATTERING
}

M. Vergnat, C. Dufour, A. Bruson, S. Houssaini, G. Marchal, Ph. Mangin, J. Rhyne, R. Erwin, C. Vettier

\section{- To cite this version:}

M. Vergnat, C. Dufour, A. Bruson, S. Houssaini, G. Marchal, et al.. MULTILAYERS AND LOW ANGLE NEUTRON SCATTERING. Journal de Physique Colloques, 1989, 50 (C7), pp.C7-207-C7212. 10.1051/jphyscol:1989722 . jpa-00229886

\section{HAL Id: jpa-00229886 https://hal.science/jpa-00229886}

Submitted on 1 Jan 1989

HAL is a multi-disciplinary open access archive for the deposit and dissemination of scientific research documents, whether they are published or not. The documents may come from teaching and research institutions in France or abroad, or from public or private research centers.
L'archive ouverte pluridisciplinaire $\mathbf{H A L}$, est destinée au dépôt et à la diffusion de documents scientifiques de niveau recherche, publiés ou non, émanant des établissements d'enseignement et de recherche français ou étrangers, des laboratoires publics ou privés. 


\title{
MULTILAYERS AND LOW ANGLE NEUTRON SCATTERING
}

\author{
M. VERGNAT, C. DUFOUR, A. BRUSON, S. HOUSSAINI, G. MARCHAL, Ph. MANGIN, \\ J.J. RHYNE*, R. ERWIN* and C. VETTIER** \\ Laboratoire de Physique du solide (UA CNRS DO 155) Université Nancy-1, \\ BP. 239, F-54506 Vandoeuvre les Nancy, France \\ * National Institute of Standards and Technology, Gaithersburg, MD \\ 20899, U.S.A. \\ * I.L.L. 156X, F-38042 Grenoble Cedex, France
}

\begin{abstract}
résumé - La diffusion des rayons $X$ et des neutrons par un système multicouches produit des " pics de Bragg " aux petits angles. L'intensité des pics est fonction du contraste entre les couches successives. Avec la technique de diffusion de neutrons, il est possible de changer le contraste et donc d'obtenir davantage d'informations sur la structure. Dans cet article, nous présentons deux exemples. Dans le premier exemple, des multicouches $\mathrm{Fe} / \mathrm{Si}$ sont étudiées par diffraction de neutrons polarisés. La variation de contraste produit par le changement de polarisation des neutrons permet de conclure à la présence d'un alliage d'interface non magnétiqué entre le Silicium amorphe et le Fer magnétique. Dans le second exemple, la modulation d' hydrogène dans des échantillons Silicium/Silicium hydrogéné est observée par substitution isotopique de $\mathrm{H}, \mathrm{D}$ et de $\mathrm{H}_{\mathrm{x}} \mathrm{D}_{1-\mathrm{x}}$.
\end{abstract}

Abstract-Multilayers give rise to "Bragg " peaks in small angle $X$-ray and neutron scattering. The intensity of the peaks is a function of the contrast between the layers. In neutron scattering experiments, information can be obtained from change of contrast between the layers or between the interfaces and the layers. In this paper two examples are presented. in the first example, iron/ silicon multilayers are studied by polarized neutron scattering. A non-magnetic alloy is found between pure iron and pure silicon. In the second example, the modulation of hydrogen in silicon/hydrogenated silicon multilayers is shown by isotopical substitution of $H, D$ and of $H_{x} D_{1-x}$ mixtures.

\section{I) Introduction}

Multilayered materials with a modulation wavelength in the $10-100 \AA$ range can valuably be studied by Low Angle X-ray Scattering (LAXS) and by Low Angle Neutron Scattering (LANS) techniques. A typical $\theta / 2 \theta$ (specular) reflectivity diagram exhibits a plateau of total reflection followed by a $(\sin \theta)^{-4}$ decrease on which "Bragg" peaks of the modulation take place. The parameters of an ideal multilayer are the thicknesses of the layers and their atomic densities. The standard deviation of the thicknesses resulting from errors in the fabrication processes, the roughness at the interfaces and the effect of alloying between the layers must be considered in real samples. In principle, all the parameters should be deduced from the whole diffraction pattern and in particular from the total reflection angle, the positions of the "Bragg" peaks, their widths, and their intensities.

The usual way to determine the parameters is to build a model of the atomic distribution, deduce the profile of scattering amplitude density, simulate the reflectivity and finally compare the calculated spectra with the experimental data. 
This simulation can be easily achieved in the dynamical theory by using the optic laws. It consists of determining the refraction indices in each medium or layer and using the Fresnel equation at the interfaces [1] [2]. The multiple reflections lead to a classical recursion formula where the roughness at the interface can be introduced by a Debye-Waller like factor [3].

The refraction indice of $X$-rays or neutrons in a medium $A$ is given by $n_{A}=1-\delta_{A}+i \beta_{A}$ where $\delta_{A}=\rho_{A}$ $\lambda^{2} / 2 \pi$. $\lambda$ is the wavelength and $\rho_{A}$ is the scattering amplitude density. $\rho_{A}$ is equal to the sum of the product of the atomic scattering amplitudes of the elements of $A$ by their atomic density in the layer. The imaginary part is related to the absorption in the layers.

The kinematical theory is considered to be valid if the reflectivity is not larger than 0.3-0.4 [4]. In this theory, the intensity of the "Bragg "peaks of an ideal multilayer is directly proportional to $\left(\rho_{A}-\rho_{B}\right)^{2}$ $|F(K)|^{2}$ where $F$ is the structure factor of the cell and $K$ the scattering vector. $\left(\rho_{A}-\rho_{B}\right)$ is the contrast between the layers. As this approximation is simple and is valid for the samples studied here, it will be used for the qualitative discussions.

Practically, the simulation method is not always direct. For example, it is difficult to distinguish between roughness and alloying at the interfaces: both lead to a damping of the specular reflectivity in the high angles range. On the other hand, with $X$-ray, it is not very convenient to see a hydrogen modulation in amorphous silicon : indeed, it is correlated with a density modulation which contributes to the diffraction pattern.

A convenient way to get more experimental information is to perform experiment in which the contrast between the layers is modified and controlled by external parameters. This can be achieved using X-ray with wavelengths close to the absorption edge of one of the elements. One can use neutrons in isotope substitution studies or one can use polarized beam when at least one of the layers is magnetic.

In this paper, we present two examples where the change of contrast has provided information that was very difficult or even impossible to obtain from one experiment and from a simple simulation.

The first example deals with the Fe/Si multilayered system. In this example iron is magnetic, silicon is non-magnetic and an alloyed interlayer is formed between pure materials. With $X$-rays or non polarized neutrons the scattering amplitude density of the interlayer has an effect equivalent to roughness. With polarized neutron it has been possible to keep the scattering amplitude density of the alloy constant and to make the scattering amplitude density of iron almost equal to that of silicon. In this case only the interface was seen.

The second example is that of silicon/hydrogenated silicon multilayers. Although the X-rays do not see the hydrogen, reflectivity peaks arise because the hydrogenated silicon is less dense that the pure silicon. In replacing the isotope $H$ by $D$ or by $a H_{x} D_{1-x}$ mixture, it was possible to show that the density modulation did correspond to the hydrogen modulation. A relation between the hydrogen content and the decrease of the silicon density has been established.

\section{II) Study of Fe/Si multilayers with polarized neutrons}

\section{Principle of the experiment}

As recalled in the introduction, the intensity of the $n^{\text {th }}$ peaks of an ideal multilayer can be written:

$$
I_{n} \sim\left(\rho S_{i}-\rho F_{e}\right)^{2} \mid F\left(K_{n}\right) I^{2}
$$

$\rho_{S i}=d_{S i} b_{S i}$ where $d_{S i}$ is the silicon density and $b_{S i}$ is the coherent scattering amplitude of silicon.

$\rho_{\mathrm{Fe}}$ depends on the magnetic state of iron and on the polarization of neutrons.

If the neutrons are not polarized, then $\rho_{\mathrm{Fe}}=\sqrt{\mathrm{b}_{\mathrm{Fe}}{ }^{2}+\mathrm{p}^{2}} \mathrm{~d} \mathrm{Fe}$.

If the neutrons are polarized parallel to the iron magnetic moments, then $\rho^{+} \mathrm{Fe}^{-}=\left(b_{\mathrm{Fe}}+p\right) \mathrm{d}_{\mathrm{Fe}}$.

If the neutrons are polarized antiparallel to the iron magnetic moments, then $\rho^{-} F e^{-}(b F e-p) d F e$.

$d_{F e}$ is the density of iron, $b_{F e}=0.97510^{-12} \mathrm{~cm}$ is the nuclear coherent scattering amplitude of iron, and $p=0,27 \mu 10^{-12} \mathrm{~cm}$ is the magnetic scattering amplitude. For $\mu=2.2 \mu_{\mathrm{B}}, \rho^{-} \mathrm{Fe}$ is very small compared to $\rho^{+} F_{e}$. The flipping ratio $R_{n}=I_{n}+I_{n}$ (ratio between intensities measured in the two geometrical configurations) is expected to be the same at all the order and is equal to $\left(\rho \mathrm{Si}^{-} \rho^{+} \mathrm{Fe}^{2}\right)^{2}\left(\rho_{\mathrm{Si}}^{-} \rho^{-} \mathrm{Fe}\right)^{2}$. From the recursion formula, the scattering amplitude profiles of an ideal multilayers pictured in figure 1a are expected to give the reflectivities shown in figure $2 a$.

\section{Experimental results}

$\mathrm{Fe} / \mathrm{Si}$ samples were prepared by evaporation of $\mathrm{Fe}$ and $\mathrm{Si}$ in a high vacuum chamber [5]. The substrates were float-glass plates and were kept at liquid nitrogen temperature during the deposition process. The evaporation rates were controlled by two independent quartz monitoring systems. The neutron scattering experiments were performed on the $\mathbb{N} 20$ instrument at the Institut Laue Langevin. The neutrons at the 
wavelengh 2.36A were polarized using a Heusler monochromator. The instrument was set in the triple axis configuration with a graphite analyser in the elastic position and a PG filter in order to reduce the background. Collimatiors were placed $10^{\circ}$ on each side of the sample. The sample was mounted in a split coil superconducting magnet, providing a vertical 2 Tesla magnetic field, in the plane of the layers. All the data were taken at $\mathbf{T}=100 \mathrm{~K}$.

As shown in figure 3 , the flipping ratio $R_{n}$ is not the same for all the peaks. It is even lower than 1 at the $4^{\text {th }}$ order. The scattering amplitude profile has to be reconsidered [6] [7] [8].
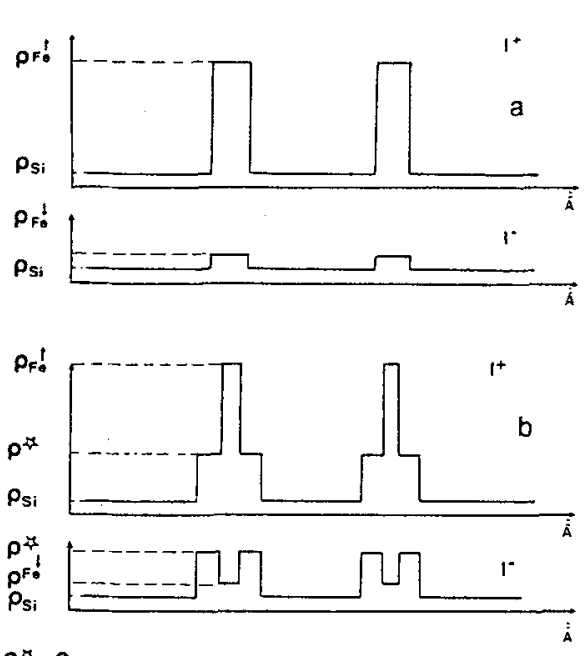

$\rho * \rho_{\text {altoy }}$

Fig.1: Schematic drawing of the neutron scattering amplitude density for a $\mathrm{Fe} / \mathrm{Si}$ multilayer ( 50 bilayers - thickness of $\mathrm{Fe}=25 \AA$ and thickness of $\mathrm{Si}=95 \AA$ )

a) abrupt profile interlayer

b)profile with a non-magnetic

(the relative scales between the atomic scattering amplitude densities are respected). $\rho^{*}$ is the scattering amplitude of the non-magnetic alloy.

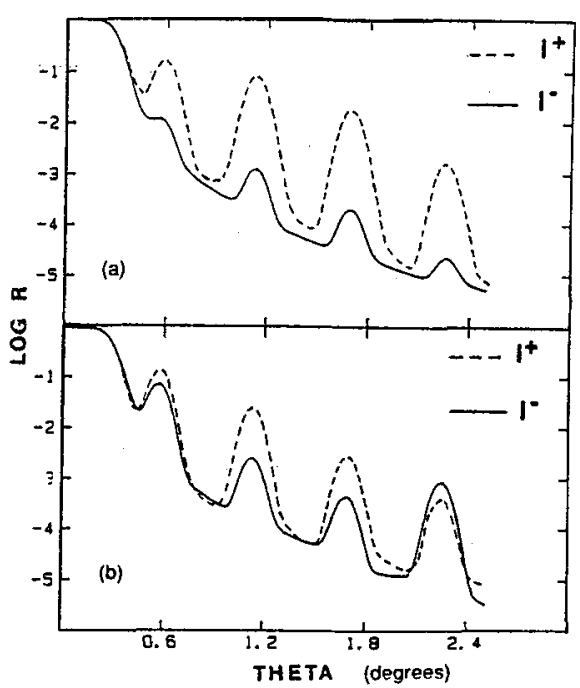

Fig.2: Calculated $\theta / 2 \theta$ polarized neutron reflectivity (from scattering amplitude profiles of Fig.1) of $\mathrm{Fe} / \mathrm{Si}$ multilayers with $\lambda=2.36 \AA$ :

a)abrupt profile

b)profile with a non-magnetic interlayer $(e=16 \AA)$

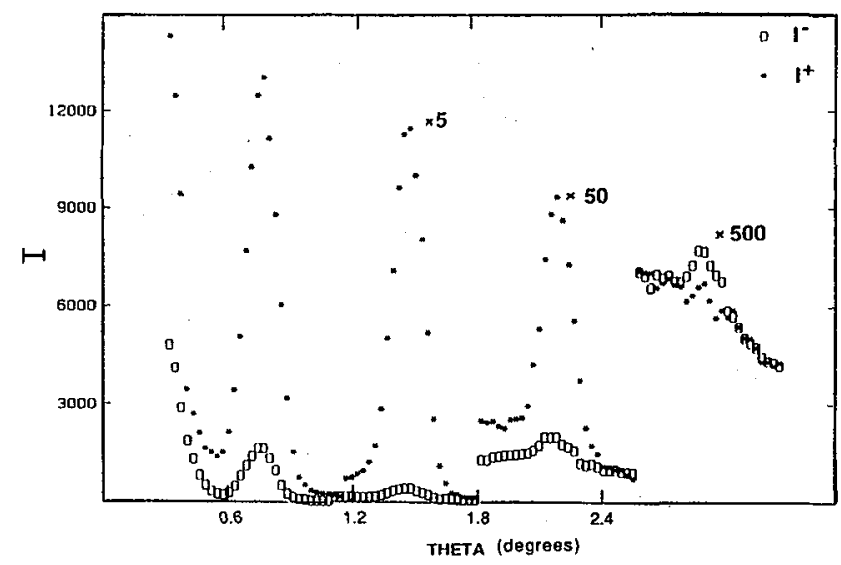

Fig.3: Experimental $\theta / 2 \theta$ polarized neutron diffraction pattern from the Fe/Si multilayer $(\lambda=2.36 \AA$ ) . $1^{+}=$intensity collected when the spins of the neutrons are parallel to the iron atomic moment.

$r=$ intensity collected in the antiparallel geometry. 
Model of atomic profile

From Mössbauer spectroscopy data [9], it was established that a part of the iron atoms of the sample was involved in an amorphous non-magnetic phase. We then postulated that this phase was located at the interface between the silicon and iron layers. In a crude hypothesis, we considered that the period was made of 4 layers: ..../Silicon/amorphous non magnetic alloy/magnetic iron/ amorphous non magnetic alloy/......The scattering amplitude density of the non magnetic alloy is $\rho^{*}$. As only the iron layers are magnetic, the scattering amplitude profiles are those pictured in figure $1 \mathrm{~b}$. Only the iron scattering amplitude changes with the neutron polarization. It is particularly interesting to note that in the antiparallel geometry the interfaces exhibit contrast with silicon layers and with iron layers. In this particular case the LANS results from the interface.

The calculated reflectivities are pictured in figure $2 \mathrm{~b}$. They reproduce the measured flipping ratios when the interlayer alloy is $16 \AA$ thick [10].

\section{III)Determination of the hydrogen profile in Si/Si:H multilayers}

\section{Preparation of the samples}

Silicon was evaporated continuously under a modulated pressure of hydrogen $(H)$, deuterium (D) or of appropriate mixtures of hydrogen and deuterium $\left(M=H_{1-x} D_{x}\right)$. The gases were introduced in the evaporation chamber through a $1 \mathrm{~mm}$ inner diameter tube heated at $2000^{\circ} \mathrm{C}$ in order to decompose hydrogen or deuterium molecules and to send a maximum of atoms to the substrate [11]. The pressure sequences were $2 \times 10^{-6}$ Torr during $30 \mathrm{~s}$ (silicon evaporated under vacuum) then $6 \times 10^{-5}$ Torr during $30 \mathrm{~s}$ (silicon evaporated under pressure of hydrogen and/or deuterium) etc... It was not possible to lower the pressure below $2 \times 10^{-6}$ Torr between two sequences of "hydrogenation". The total number of layers was 100, and the expected deposition rate of silicon on the substrates was about $1.3 \AA / \mathrm{s}$. The samples were removed at room temperature for measurements. Two types of sequences were grown:

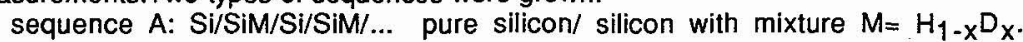

sequence B: $\mathrm{Si} / \mathrm{SiH} / \mathrm{Si} / \mathrm{SiD} / .$. same kind of sequence, but the isotope was alternately hydrogen or deuterium

\section{Electron microscopy and low angle $X$-ray scattering}

By using a microcleavage technique [12], some fragments of the samples were collected on an electron microscope grid. The cross sectional micrographs show a sequence of dark and light stripes (fig.4).

The low angle X-ray scattering (LAXS) patterns collected from samples of sequences $A$ and $B$ are identical. They exhibit two diffraction peaks. showing the presence of a structural modulation (Fig.5). The wavelength of the modulation (i.e., the width of the bilayers), as calculated from the position of the peaks, is $90 \AA$, which is significantly larger than the modulation expected from a deposition in high vacuum, $80 \AA$.

As the electron and $X$-ray scattering from hydrogen and deuterium are very weak, they are not the origin of the contrast. More likely, the contrast originates in the modulation of the density of silicon which is known to be reduced when silicon is evaporated under hydrogen pressure [13].

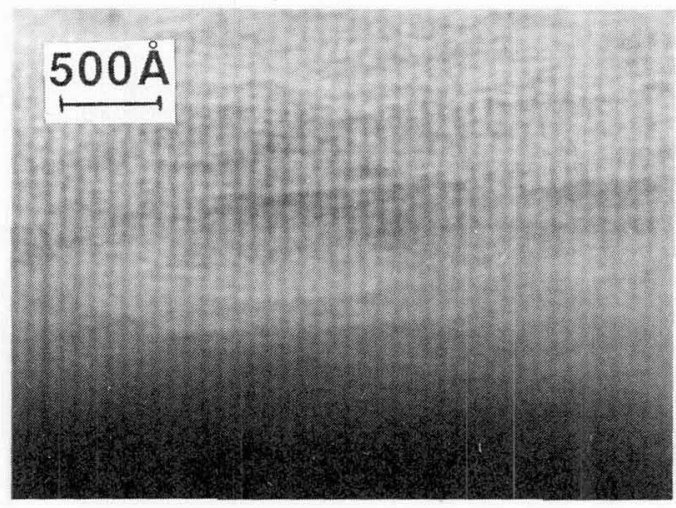

Fig.4: Cross-sectional electron micrograph of Si/Si:H/Si/Si:H multilayers

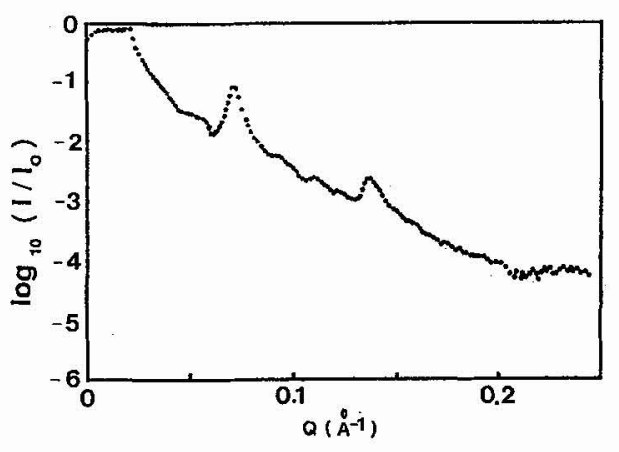

Fig.5: Low angle $X$-ray scattering intensity versus scattering vector $q$ for multilayers of type Si/Si:M/Si/Si:M $\left(M=H_{x} D_{1-x}\right)$. 


\section{Low angle neutron scattering}

As the $H$ and $D$ scattering amplitude of neutrons are very different and of opposite sign, $b_{H}=-0.374 \times 10^{-}$ $12 \mathrm{~cm}$, and $\mathrm{b}_{\mathrm{D}}=0.667 \times 10^{-12} \mathrm{~cm}$, the hydrogen modulation can be shewn by changing the hydrogen contrast. Neutron diffraction patterns from type $A$ samples exhibit one diffraction peak at $q=0.070 \AA^{-1}$. This corresponds to the first LAXS peak. However, the intensity of this peak varies considerably with $x$. As shown in figure 6 , it is maximum for $x=0(M=H)$, decreases with $x$, reaches 0 for $x=0.7$ and increases again when $x$ is larger than 0.7 . It is interesting to note that the intensity of this peak is not zero when the average coherent scattering of $M(x=0.36)$ is zero.

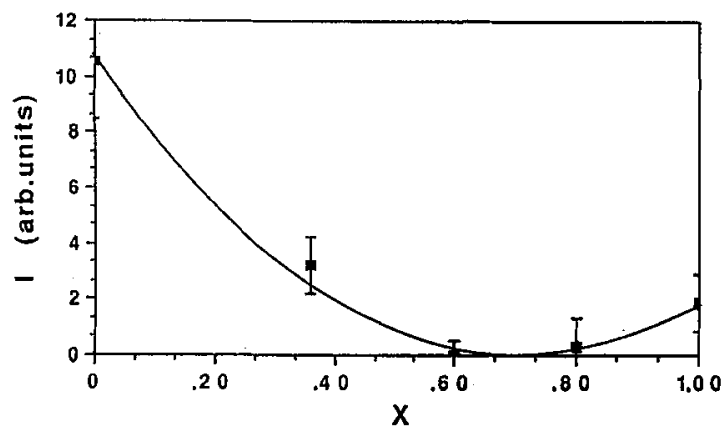

Fig.6: Intensity of the first diffraction peak versus concentration $x$ of the $H_{1-x} D_{x}$ mixture

- experimental data

- parabolic fit

The interpretation of the data is straightforward in the kinematical approximation if we consider both the silicon density and the hydrogen content in the determination of the coherent scattering amplitude density. As illustrated in Fig.7, the contrast is maximum when $\mathrm{M}=\mathrm{H}$. The negative coherent scattering amplitude of $\mathrm{H}$ adds to the loss of silicon density to enhance the contrast. When $M=H_{0.64} D_{0.36}, b_{M}=0$ and the contrast is only due to the difference of silicon density in the sublayers. For $M=H_{0.40} D_{0.60}, \rho_{A}$ is very close to $\rho_{B}$ and the contrast between the sublayers is very poor. The coherent scattering amplitude of $M$ compensates the loss of the silicon density. Finally for $M=D, \rho_{B}$ becomes larger than $p_{A}$ and the contrast reappears. In this model, the intensity of the peak is proportionnal to $d_{S i}{ }^{2}\left[b_{S i} t-\left(x_{D}+(1-x) b_{H}\right) C\right]^{2} \cdot t$ is the relative loss of silicon atomic density in the hydrogenated silicon layers and $\mathrm{C}$ is the atomic percentage of hydrogen atoms in the same layers. The intensity varies with $x$ as a parabola. The minimum of the best fitting parabola, located at $x=0.7$, leads to the relation: $C \simeq 1.2 \mathrm{t}$ which links the loss of density of silicon to the hydrogen content.

LANS patterns from type B samples show again a first peak attributed to the silicon density modulation corresponding to that observed in low angle X-ray scattering. But, in addition, a large pre-peak at $q=0.036$ $A^{-1}$ is found. In this case, the neutrons see the period $\rho_{S i}, \rho_{S i}: H, P_{S i}, \rho_{S i}$. The wavelength of the contrast is doubled and a superstructure peak appears.

The above parabolic evolution of the intensity and the superstructure peak clearly show the hydrogen modulation and its connection with the silicon density variation.

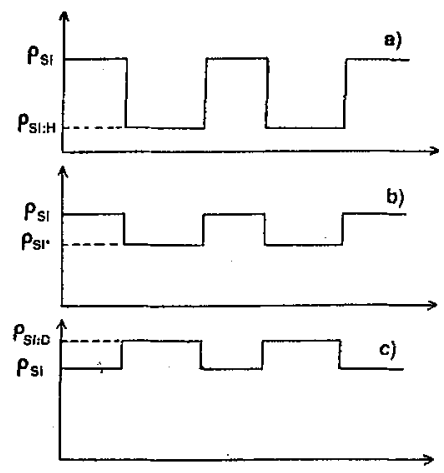

Fig.7: Schematic drawing of the neutron coherent scattering amplitude density $\rho$ for:

a) multilayers $\mathrm{Si} / \mathrm{Si}: \mathrm{H} / \mathrm{Si} / \mathrm{Si} \mathrm{H}$

b) multilayers $\mathrm{Si} / \mathrm{Si}: \mathrm{M} / \mathrm{Si} / \mathrm{Si} \mathrm{M}$ ( $\mathrm{M}$ is

the zero mixture $\left.H_{.64} D_{.36}\right) \rho_{S i}{ }^{*}=\rho_{S i: M}$

c) multilayers $\mathrm{Si} / \mathrm{Si}: \mathrm{D} / \mathrm{Si} / \mathrm{Si}: \mathrm{D}$ 


\section{IV) Conclusion}

From the two examples it has been shown that the low angle neutron scattering technique can be a very powerful tool for the determination of multilayered structure. Its applications are not restricted to the study of static structure. It is to use for the determination of the diffusion process of hydrogen in silicon or for the study of magnetic transition in mutilayers.

Acknowledgment:This work was supported by Nato collaborative research grants $N^{\circ} 550 / 87$.

References:

[1] UNDERWOOD, J.H. and BARBEE, T.W., Appl. Opt. 20 (1981) 3027.

[2] VIDAL, B. and VINCENT,P., Appl. Opt. 23 (1984) 1794.

[3] NEVOT, L., PARDO, B. and CORNO, J., Rev. Phys. Appl. 23 (1988) 1675.

[4] SEARS, V.F., Acta Crystallogr. A39 (1983) 601.

[5] MARCHAL, G., MANGIN, Ph. and JANOT, C., Phil. Mag. 32 (1975) 1007.

[6] MAJKRZAK, C.F., AXE, J.D. and BÖNI, P., J. Appl. Phys. 573 (1985) 3657.

[7] MAJKRZAK, C.F., Physica 136B (1986) 69-74.

[8] MEZEl, F., Summer school "Physics, fabrication and application of multilayers structures" Bandor, France (July 1987).

[9] DUFOUR, C., BRUSON, A., GEORGE, B., MANGIN, Ph. and MARCHAL, G., Proceedings of I.C.M.88, J. Phys. 49 Colloque C.8 Suppl. 12 (1988) 1781.

[10] DUFOUR, C., BRUSON, A., GEORGE, B., MANGIN, Ph., MARCHAL, G., RHYNE, J.J., ERWIN, R. and VETTIER, C., Solid State Commun. 69 (1989) 963.

[11] VERGNAT,M., MARCHAL,G. and PIECUCH, M., Revue Phys. Appl., 22 (1987) 1803.

[12 ] LEPETRE, Y. and RASIGNI, G. Opt. Lett., 9(1984)433.

[13] VERGNAT,M., Thesis (1988) Nancy; France. 